\title{
Frustrated Synchronization in Two Coupled Polygonal Oscillatory Networks
}

\author{
Yoko Uwate and Yoshifumi Nishio \\ Dept. of Electrical and Electronic Engineering, \\ Tokushima University \\ 2-1 Minami-Josanjima, Tokushima, Japan \\ Email: \{uwate, nishio\}@ee.tokushima-u.ac.jp
}

\begin{abstract}
In this study, synchronization phenomena observed in coupled polygonal oscillatory networks with frustration is investigated. We focus on the amplitude of each oscillator when the coupling strength is changed. By using computer simulations and circuit experiments, we confirm that the amplitude of the shared and the other oscillators obtains different value by increasing the value of the coupling strength. Furthermore, theoretical analysis is applied to solve the amplitude and the phase differences between the adjacent oscillators.
\end{abstract}

\section{INTRODUCTION}

Synchronization phenomena with coupled oscillators are suitable model to analyze the natural phenomena. Therefore, many researchers have proposed different coupled oscillatory networks and have discovered many interesting synchronization phenomena [1]-[4].

On the other hand, there are several types of polygonal network structures (e.g. honeycomb, soup bubbles and cracks of drying mud structure) in the natural science. Generally, for the studies of large-scale network using coupled oscillators, a ring, a ladder and a two dimensional array structure are often investigated. However, there are not many discussions about coupled polygonal oscillatory networks by using electrical oscillators.

In our research group, we focus on synchronization phenomena of coupled oscillators under a difficult situation for the circuit. Setou et al. have reported the synchronization phenomena in $N$ oscillators coupled by resistors as a ring. The oscillation stop in some range of the coupling resistors was confirmed [5]. We have investigated synchronization phenomena in coupled polygonal oscillatory networks sharing branches [6]. In this system, van der Pol oscillators are connected to every corner of polygonal network. By using computer simulations and theoretical analysis, we confirm that coupled oscillators tend to synchronize to minimize the power consumption of the whole system. Furthermore, the phase difference of the shared oscillators is solved by finding the minimum value of the power consumption function.

However, we have not discussed synchronization state of the proposed oscillatory networks by changing the coupling parameter. In this study, synchronization phenomena observed in coupled polygonal oscillatory networks with frustration is investigated. We focus on the amplitude of each oscillator when the coupling strength is changed. By using computer simulations and circuit experiments, we confirm that the amplitude of the shared and the other oscillators obtains different value by increasing the value of the coupling strength. Furthermore, theoretical analysis is applied to solve the amplitude and the phase differences between the adjacent oscillators. We expect that the results of this study contribute to understanding of synchronization phenomena observed in general complex networks.

\section{Two Coupled Oscillatory Networks}

First, we investigate the synchronization phenomena in two coupled oscillatory systems. Two identical polygonal oscillatory networks are coupled by sharing a branch as shown in Fig. 1. In this circuit model, we consider the coupling method which two adjacent oscillators are tend to synchronize at anti-phase state. We call the first and the second oscillators which are connected to both side of polygonal network "shared oscillators."

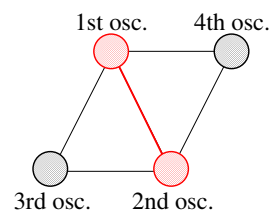

(a) 3-3 coupling network

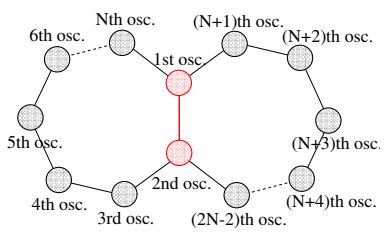

(b) $N-N$ coupling (general) network
Fig. 1. Two coupled polygonal oscillatory networks.

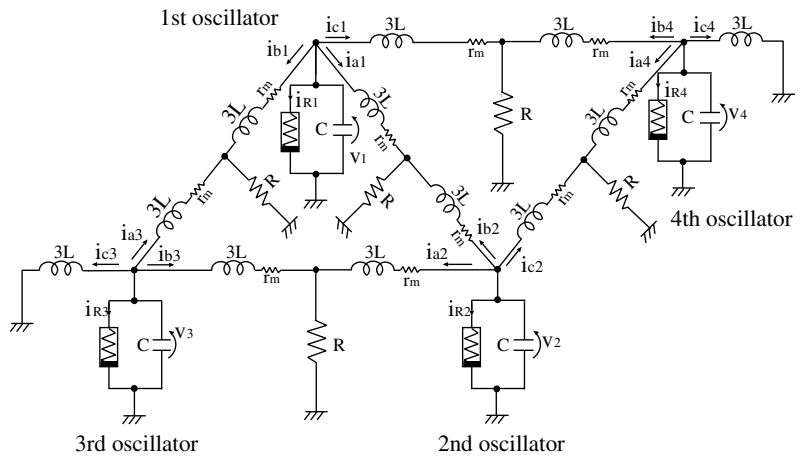

Fig. 2. Circuit model for $3-3$ coupling oscillatory networks.

Next, we develop the expression for the circuit equations of $3-3$ coupling oscillatory networks as shown in Fig. 2. The 
$v_{k}-i_{R k}$ characteristics of the nonlinear resistor are approximated by the following third order polynomial equation,

$$
i_{R k}=-g_{1} v_{k}+g_{3} v_{k}{ }^{3} \quad\left(g_{1}, g_{3}>0\right),(k=1,2,3,4) .
$$

The normalized circuit equations governing the circuit are expressed as

[ $k$ th oscillator]

$$
\left\{\begin{aligned}
& \frac{d x_{k}}{d \tau}=\varepsilon\left(1-\frac{1}{3} x_{k}^{2}\right) x_{k}-\left(y_{a k}+y_{b k}+y_{c k}\right) \\
& \frac{d y_{a k}}{d \tau}=\frac{1}{3}\left\{x_{k}-\eta y_{a k}-\gamma\left(y_{a k}+y_{n}\right)\right\} \\
& \frac{d y_{b k}}{d \tau}=\frac{1}{3}\left\{x_{k}-\eta y_{b k}-\gamma\left(y_{b k}+y_{n}\right)\right\} \\
& \frac{d y_{c k}}{d \tau}=\frac{1}{3}\left\{x_{k}-\eta y_{c k}-\gamma\left(y_{c k}+y_{n}\right)\right\} \\
&(k=1,2,3,4) .
\end{aligned}\right.
$$

In this equations, $\gamma$ is the coupling strength, $\varepsilon$ denotes the nonlinearity of the oscillators and $y_{n}$ denotes the current of neighbor oscillator on coupling resistor.

\section{A. Synchronization Phenomena}

Figure 3 shows the attractor of each oscillator in 3-3 coupling network when the coupling strength is changed. First, the amplitudes of the shared oscillators are decreased (Fig. 3(B)). By increasing the coupling strength, torus attractors are observed (Figs. 3(C), (D)). Poincare maps of torus attractors are shown in Fig. 4.

Figures 5 and 6 show the time wave form of the voltage charged at the capacitance of each oscillator obtained from the computer simulation. From this figure, we can see that the first and the second oscillators are synchronized at in-phase (phase difference: 0 degree). While, the other combination oscillators synchronize with anti-phase (phase difference: 180 degree).
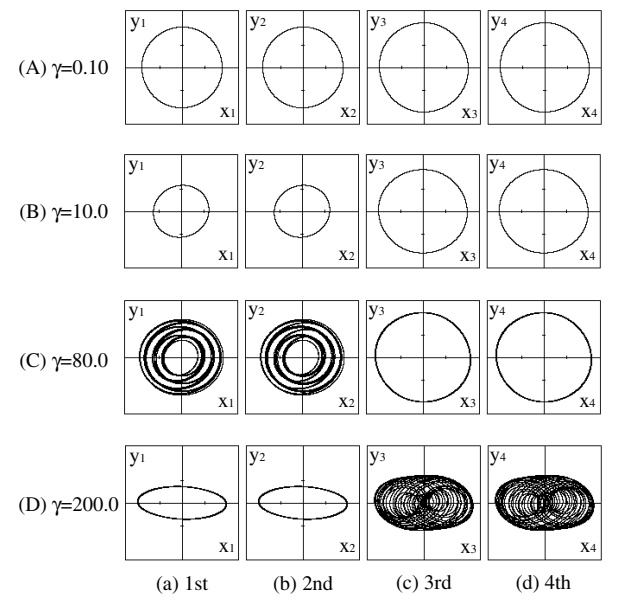

Fig. 3. Attractors observed in 3-3 coupling network.

We also obtain the same synchronization states from the circuit experiment as shown in Figs. 7, 8. The circuit experiments parameters are set to $L=50 \mathrm{mH}$ and $C=33 n F$. The nonlinear resistor is realized by op-amp (TL082). The power supply of the op-amp is fixed as $\pm 12 \mathrm{~V}$.

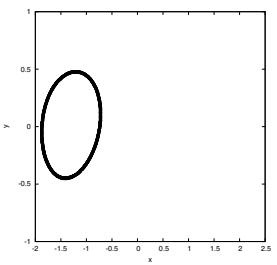

(a) 1st oscillator $(\gamma=80.0)$

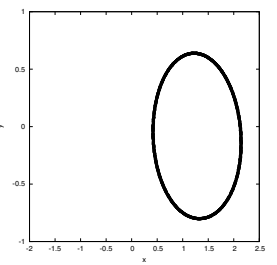

(b) 3rd oscillator $(\gamma=200.0)$

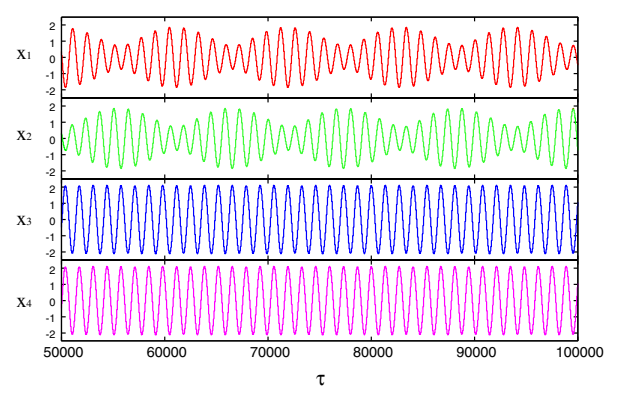

Fig. 5. Time wave forms of 3-3 coupling network $(\gamma=80.0)$.

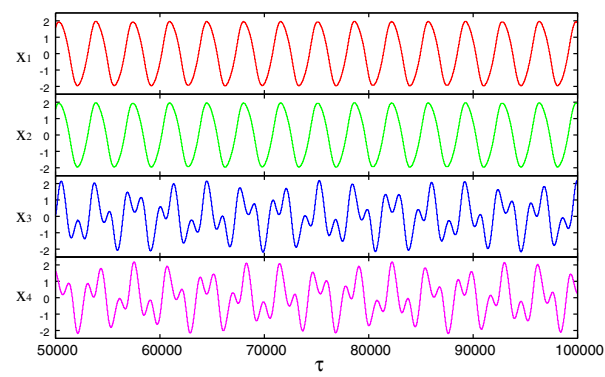

Fig. 6. Time wave forms of 3-3 coupling network $(\gamma=200.0)$

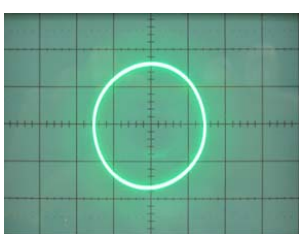

(a) One periodic attractor

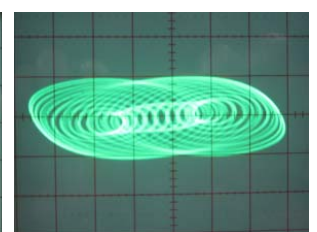

(b) Torus attractor.
Fig. 7. Attractors from circuit experiments.

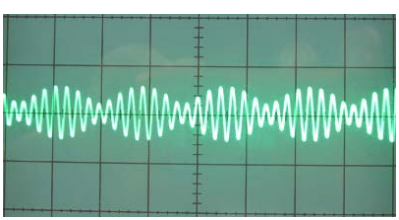

(a) $v_{1}(R=5.1 \mathrm{k} \Omega)$.

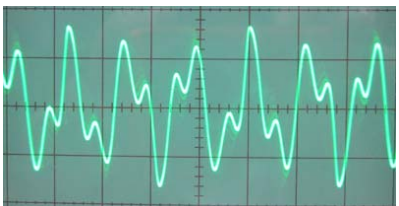

(b) $v_{3}(R=30.0 \mathrm{k} \Omega)$.

Fig. 8. Time wave forms of torus from circuit experiments. 


\section{B. One Parameter Bifurcation}

The one parameter bifurcation diagram of $x_{1}$ and $x_{3}$ is shown in Fig. 9. From this figure, we can confirm that the amplitudes of $x_{1}$ and $x_{3}$ have similar value when the coupling strength is smaller than $\gamma=0.02$. Around $\gamma=0.1$, the amplitude of $x_{1}$ decreases and $x_{3}$ keeps the amplitude almost 2.0. By increasing $\gamma$, the both of the amplitudes start to decrease and take extreme value around $\gamma=1.5$. After that the both of the amplitudes increase until $\gamma=50.0$. The torus attractor can be observed in 1st oscillator $\left(x_{1}\right)$ from $\gamma=50.0$ to 100.0. When $\gamma$ reaches 100.0 , the 1 st oscillator switches one periodic attractor and the 3rd oscillator generates torus attractor.

Generally, oscillation death can be occurred in symmetrical coupled oscillatory system by increasing the coupling strength. However, the oscillation death can not be observed in this circuit system. We consider that the unbalanced circuit structure affect to synchronization state materially.

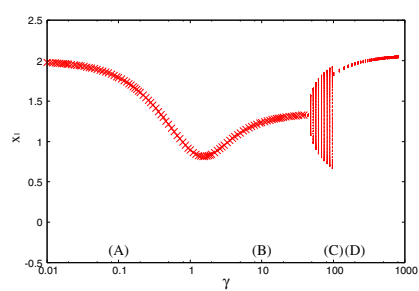

(a) $x_{1}$.

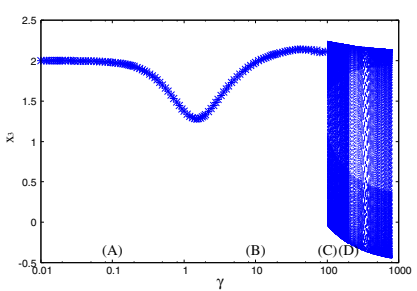

(b) $x_{3}$.
Fig. 9. One parameter bifurcation.

Figure 10 shows the one parameter diagram of the phase difference. The shared oscillators are synchronized at in-phase and the other combination oscillators are synchronized at antiphase when the coupling strength is smaller than $\gamma=100.0$. The all coupled oscillators synchronize with in-phase if $\gamma$ is larger than 100.0 .

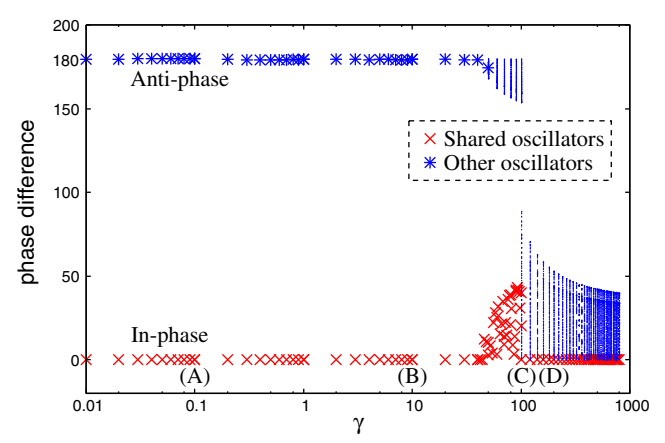

Fig. 10. Phase difference.

\section{Theoretical Analysis}

In this section, we analyze the synchronization phenomena by using the averaging method which is one of the theoretical analysis.

First, consider the case of that the tiny resistors to avoid $L$ loop for computer simulation is set to zero $(\eta=0.0)$ in Eq. (2). We assume that 1 st and 2 nd oscillators are same amplitude and phase difference by symmetrical circuit structure. Similarly, 3rd and 4th oscillators are same characteristics. Equation (2) is combined to give the second order nonlinear differential equation as follows,

$$
\begin{aligned}
& \frac{d^{2} x_{k}}{d \tau^{2}}+x_{k}=\varepsilon\left(1-x_{k}{ }^{2}\right) \frac{d x_{k}}{d \tau}+ \frac{1}{3} \gamma Y_{k} \equiv F_{k} \\
&(k=1,2,3,4)
\end{aligned}
$$

where

$$
\begin{gathered}
Y_{1} \equiv y_{a 1}+y_{b 1}+y_{c 1}+y_{a 3}+y_{b 2}+y_{b 4}, \\
Y_{3} \equiv y_{a 3}+y_{b 3}+y_{a 2}+y_{b 1} .
\end{gathered}
$$

Let us assume the solutions of Eq. (2) is

$$
x_{k}(\tau)=\rho_{k} \sin \left(\tau+\theta_{k}\right) .
$$

We pay attention to treat the non-resonance system and apply for the averaging method to Eq. (2). The average method is defined as following equations.

$$
\begin{aligned}
& \dot{\rho_{k}}=\lim _{T \rightarrow \infty} \int_{0}^{T} F_{k} \cos \left(\tau+\theta_{k}\right) d \tau \\
& \dot{\theta_{k}}=\lim _{T \rightarrow \infty} \int_{0}^{T} \frac{1}{\rho_{k}} F_{k} \sin \left(\tau+\theta_{k}\right) d \tau
\end{aligned}
$$

By solving the above equations, the phase difference and the amplitude are obtained as follows.

Phase differences:

$$
\begin{aligned}
& \theta_{1}-\theta_{2}=0 . \\
& \theta_{2}-\theta_{3}=\theta_{3}-\theta_{1}=\pi .
\end{aligned}
$$

Amplitudes:

$$
\begin{aligned}
& \dot{\rho_{1}}=\frac{\varepsilon \rho_{k}}{8}\left(\rho_{k}^{2}-4\right)+\frac{\gamma}{2\left(4 \gamma^{2}+9\right)}\left(-4 x_{1}+x_{3}\right) . \\
& \dot{\rho_{3}}=\frac{\varepsilon \rho_{k}}{8}\left(\rho_{k}^{2}-4\right)+\frac{\gamma}{2\left(4 \gamma^{2}+9\right)}\left(-2 x_{3}+x_{1}\right) .
\end{aligned}
$$

In the steady state, $\dot{\rho_{k}}=0$ must be satisfied. By using Newton method for Eqs. (7) and (8), the amplitudes of $\rho_{1}$ and $\rho_{3}$ are solved. In order to confirm the credibility of theoretical analysis, we compare the amplitude of oscillator of the theoretical result with the computer simulation result (see. Fig. 11). From these results, we confirm that they match very well when the coupling strength $\gamma$ is smaller than 1.5. We consider that the error between the theoretical and simulation results is occurred by harmonic component when the coupling strength $\gamma$ is larger than 1.5.

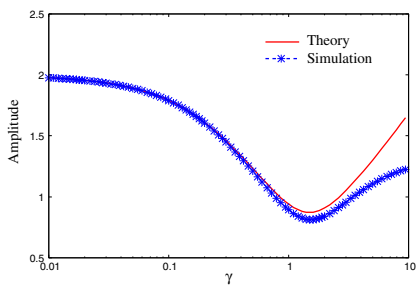

(a) $\rho_{1}$.

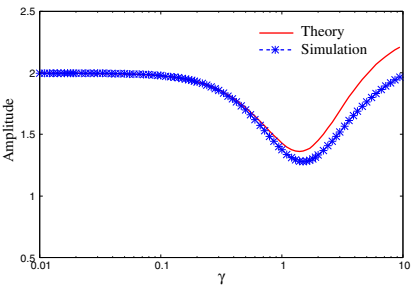

(b) $\rho_{3}$.
Fig. 11. Results of theoretical analysis. 


\section{7-7 COUPLing OSCILlATORY NETWORKS}

In this section, we consider 7-7 coupling networks. The attractors are summarized in Fig. 13 when the coupling strength is fixed with $\gamma=10.0,50.0$ and 80.0. In the case of $\gamma=10.0$, the all of oscillators generate one periodic attractors as shown in Fig. 13(a). When $\gamma$ equals 50.0, the oscillation death can be observed in 5th and 10th oscillators which are connected most far place from the shared oscillators (see Fig. 13(b)). By increasing the value of $\gamma$, the 5th and the 10th oscillators start to oscillate again (see Fig. 13(c)).

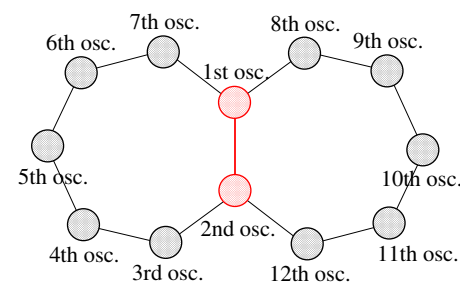

Fig. 12. 7-7 coupling network.

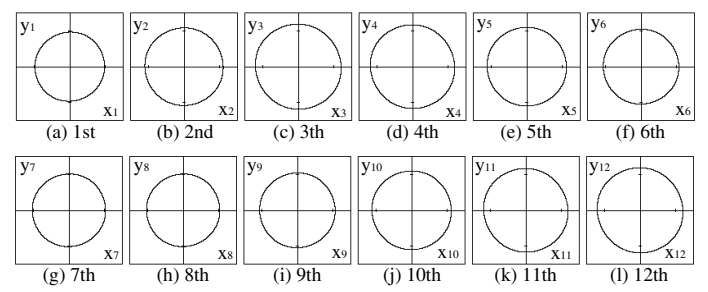

(a) $\gamma=10.0$

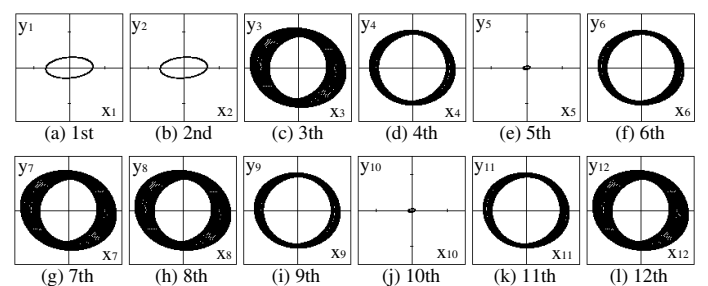

(b) $\gamma=50.0$

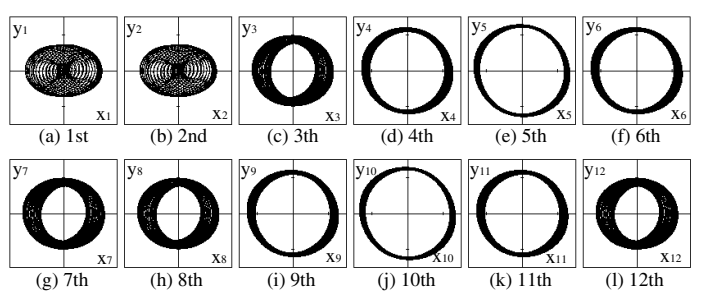

(c) $\gamma=80.0$

Fig. 13. Attractors observed in 7-7 coupling network.

\section{Extension to General Network}

Finally, we consider the bubble structure as one example of general nature patterns. The bubble structure is shown in Fig. 14(a). In this model, four different size of bubbles are connected. Ten oscillators are coupled depending on the size of bubble.
Figure 14(b) shows the amplitudes of each oscillator in the bubble network. We confirm that all amplitude have similar value when $\gamma$ is smaller than 0.002. By increasing the coupling strength, each amplitude has different value. One example of attractors in the bubble network is shown in Fig. 15.

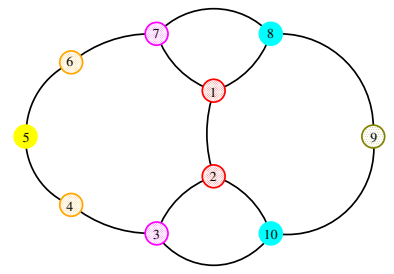

(a) Bubble structure

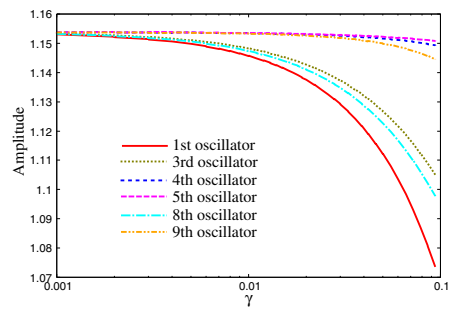

(b) Amplitude.
Fig. 14. Conceptual circuit model of bubble structure and result of amplitude.

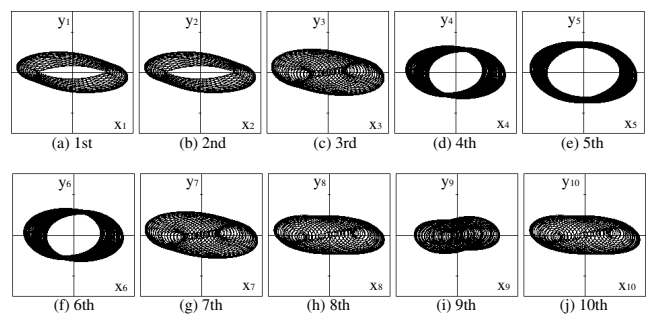

Fig. 15. Attractors of bubble network $(\gamma=400.0)$.

\section{CONCLUSION}

In this study, synchronization phenomena observed in coupled polygonal oscillatory networks with frustration is investigated. We focus on the amplitude of each oscillator when the coupling strength is changed. By using computer simulations and circuit experiments, we confirm that the amplitude of the shared and the other oscillators obtains different value by increasing the value of the coupling strength. Furthermore, theoretical analysis is applied to solve the amplitude and the phase differences between the adjacent oscillators.

\section{ACKNOWLEDGMENT}

This work was partly supported by JSPS Grant-in-Aid for Young Scientists 23700269.

\section{REFERENCES}

[1] M.G. Rosenblum, A.S. Pikovsky, and J. Kurths, "Phase Synchronization of Chaotic Oscillators" Physical Review Letters, vol.76, no.11, pp.18041807, Mar. 1996.

[2] W. Wang, I.Z. Kiss, and J.L. Hudson, "Experiments on Arrays of Globally Coupled Chaotic Electrochemical Oscillators: Synchronization and Clustering" Chaos, vol.10, no.1, pp.248-256, Mar. 2000.

[3] M. Yamauchi, Y. Nishio and A. Ushida, "Phase-waves in a ladder of oscillators" IEICE Trans. Fundamentals, vol.E86-A, no.4, pp.891-899, Apr. 2003

[4] H.B. Fotsina and J. Daafouza, "Adaptive synchronization of Uncertain Chaotic Colpitts Oscillators based on Parameter Identification" Physics Letters A, vol.339, pp.304-315, May 2005.

[5] Y Setou, Y Nishio and A. Ushida "Synchronization Phenomena in Many Oscillators Coupled by Resistors as a Ring," Proc. of APCCAS'94, pp. 570-575, Dec. 1994.

[6] Y. Uwate and Y. Nishio, "Synchronization in Several Types of Coupled Polygonal Oscillatory Networks," IEEE Trans. Circuits Syst. I, vol. 59, no. 5, pp. 1042-1050, May 2012. 